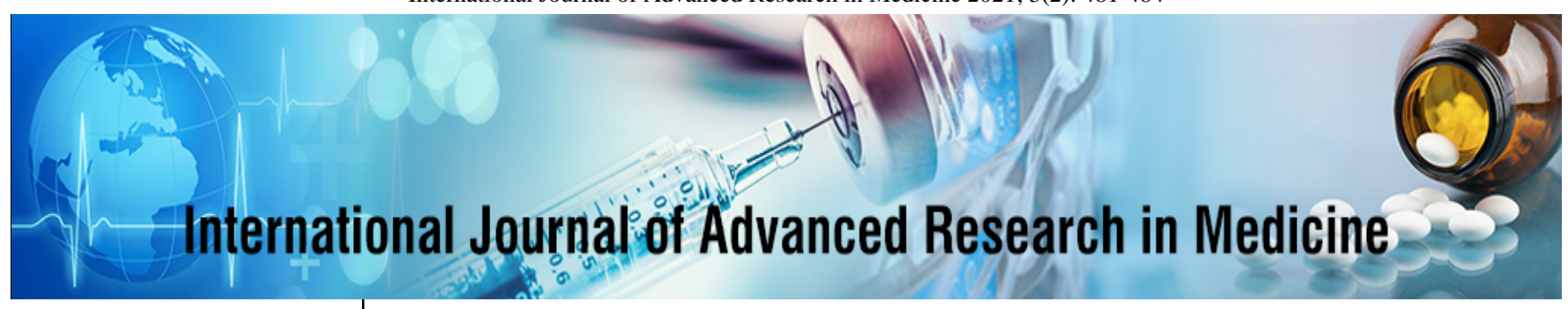

E-ISSN: 2706-9575

P-ISSN: 2706-9567

IJARM 2021; 3(2): 481-484

Received: 21-05-2021

Accepted: 05-07-2021

\section{R Kiruthika}

Post Graduate, Department of General Medicine, Rajah

Muthiah Medical College and

Hospital, Chidambaram,

Tamil Nadu, India

\section{R Umarani}

Professor, Department of General Medicine, Rajah Muthiah Medical College and Hospital, Chidambaram,

Tamil Nadu, India

\section{A Nanjilkumaran}

Assistant Professor,

Department of General

Medicine, Rajah Muthiah

Medical College and Hospital,

Chidambaram, Tamil Nadu,

India

\title{
Prevalence of raised cardiac markers in SVT and variables associated with raised troponin I
}

\author{
R Kiruthika, R Umarani and A Nanjilkumaran
}

DOI: https://doi.org/10.22271/27069567.2021.v3.i2h.293

\begin{abstract}
Cardiac troponins are the major sensitive and specific markers of myocardial injury. Joint European Society of Cardiology/American College of Cardiology committee has given the redefinition of myocardial infarction (MI) stating that troponins are the preferred cardiac markers for detecting myocardial injury. Besides MI, elevated troponins are also seen in tachyarrhythmias, acute cerebrovascular accident, acute pulmonary thromboembolism, chronic kidney disease, septic shock, myocardial injury. Though Troponin I frequently increases during myocardial injury, the etiology may vary from coronary to non- coronary causes.

Paroxysmal supraventricular tachycardia (PSVT) is the commonest form of arrhythmia seen in the Emergency Department. In contrast to ventricular tachyarrhythmias these patients are haemodynamically stable and they have good prognosis on treatment. Serum cardiac troponin I (cTnI) testing provides momentous risk stratification in these group of arrhythmias. This study will provide comprehensive data on prevalence, clinical profile, risk factors and prognosis of patients with elevated Troponin I in SVT in non CAD patients.
\end{abstract}

Keywords: Troponin I, non CAD, SVT, Prevalence.

\section{Introduction}

More than one third of the patients with Supraventricular Tachycardia (SVT) present with chest pain. It is unclear if Troponin markers in the current setting are useful in predicting which patients will have Coronary Artery Disease (CAD). In fact, modest Troponin elevation is well documented in patients with SVT with normal coronary angiography and is thought to be due to cardiac stretch, poor diastolic perfusion, and/or coronary artery vasospasm ${ }^{[1]}$. In virtue of elevated Troponin levels in patients with SVT in the present clinical era, we sought to determine the prognostic value of Troponin assays in patients presenting to the emergency department with SVT.

Recent studies have indicated that $48 \%$ of patients have elevated Troponins after SVT ${ }^{[2-4]}$. Current literature also states that more than two third of the patients with evidence of myocardial ischemia/injury by ST segment depression and Troponin I increase during SVT did not have obstructive Coronary Artery Disease ${ }^{[5]}$. Momentarily there is no difference in the diagnosis of Coronary Artery Disease based on Troponin elevation. However the elevated Troponin levels with SVT were associated with increased risk of mortality, defined by death, myocardial infarction or cardiovascular rehospitalization ${ }^{[6]}$. Furthermore, most of the patients with SVT and elevated Troponins were found to have angiographically normal coronaries $^{[1]}$.

This study will provide comprehensive data to determine the predictive value of elevated troponins in patients with SVT having persistent symptoms after conversion to normal sinus rhythm, abnormal electrocardiogram after conversion to normal sinus rhythm or those having significant risks and pre-test probability for underlying coronary artery disease.

\section{Aims and Objectives}

Corresponding Author:

R Umarani

Professor, Department of General Medicine, Rajah Muthiah Medical College and Hospital, Chidambaram, Tamil Nadu, India
- To study the prevalence of Troponin I elevation in patients presenting with Supraventricular tachycardia.

- To study the multivariate predictors of SVT such as age, chest pain at presentation, low diastolic blood pressure and longer duration of tachycardia.

- To study the heart rate during SVT and the presence of impaired left ventricular systolic function. 


\section{Materials and Methodology}

Type of study: Prospective study

Time frame of study: September 2019 to October 2021

The study was conducted in all patients presenting with supraventricular tachycardia, admitted in the Intensive care units, outpatient departments and other wards of RMMCH.

\section{Inclusion Criteria}

All patients presented with

- Signs and symptoms consistent with a diagnosis of Supraventricular tachycardia.

- Regular narrow- complex tachycardia within 4 hours.

\section{Exclusion Criteria}

1. Patients less than 18 years of age.

2. Pregnancy

3. Not willing for consent

4. Patient with poor echo window (COPD, EMPHYSEMA, OBESE)

5. Severe anemia $(\mathrm{Hb}<8 \mathrm{gm} / \mathrm{dl})$

6. Hemodynamically significant valvular heart disease

7. Prosthetic valve replacement

8. Ventricular pacemaker

9. Patients with history or current evidence of Sepsis, Pulmonary embolism, Renal failure, Heart failure, Pericarditis, Stroke/Intracranial bleed, Takotsubo cardiomyopathy, wide-complex tachycardia and following angioplasty, endurance/exercise, cardiac contusion and chemotherapy.

\section{Our study}

We prospectively enrolled 50 patients (age $>18$ years) who were admitted to the Intensive care units and other wards of RMMCH within 4 hours after the onset of a regular narrowcomplex tachycardia, with symptoms of chest pain or dyspnea after excluding exclusion criteria. During hospitalisation few patients responded to Carotid sinus massage and valsalva maneuver, while rest of the patients recovered with a single dose of Adenosine.

\section{Statistical Analysis}

Continuous variables are described as mean \pm Standard Deviation or median and interquartile range, and categorical variables are described as number and percentage. Continuous variables were compared between patients with and without hs-cTnI elevation, with use of the Student $t$ or Mann-Whitney U test. Categorical variables were compared between the mentioned groups by using the $\chi^{2}$ or Fisher exact test. ${ }^{*} \mathrm{P}$ values $<0.05$ were considered statistically significant.

\section{Results}

\section{Demographic Profile}

Table 1: Distribution of Study Participants According to Age Group (n-50)

\begin{tabular}{|c|c|c|}
\hline Age group (years) & Frequency & Percentage (\%) \\
\hline $16-30$ & 13 & 26 \\
\hline $30-60$ & 23 & 46 \\
\hline$>60$ & 14 & 28 \\
\hline Total & 50 & $100 \%$ \\
\hline
\end{tabular}

The age of the study participants range from 16-80 years. Mean age of the study participants was $45.68 \pm 18.17$ years.

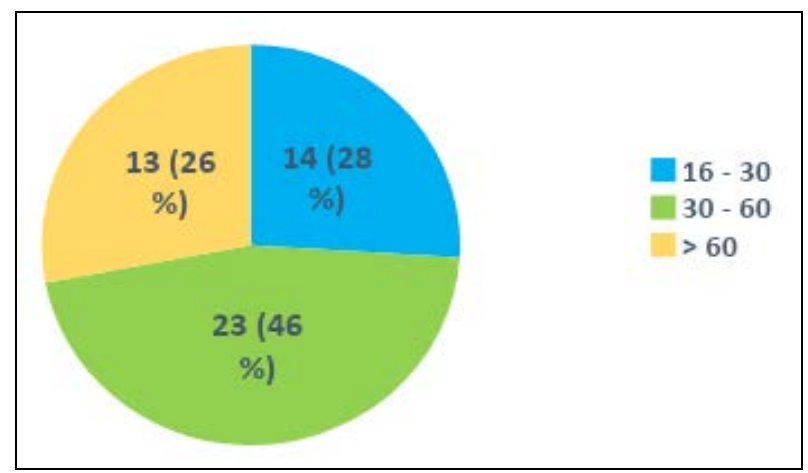

Fig 1: Distribution of study participants according to age group (in years) $(n=50)$

Table 2: Distribution of study Participants According to Abnormal ECHO Findings ( $N=23)$

\begin{tabular}{|c|c|c|}
\hline Abnormal Echo findings & Frequency & Percentage (\%) \\
\hline AV Sclerosis, Grade 1 DD & 1 & 4.3 \\
\hline Grade 3 DD & 1 & 4.3 \\
\hline AV Sclerosis, LVH & 1 & 4.3 \\
\hline LVH, Grade 1 DD & 1 & 4.3 \\
\hline Grade 1 DD & 2 & 8.7 \\
\hline Grade 2 DD & 2 & 8.7 \\
\hline LVH & 2 & 13.1 \\
\hline Grade 1 SD & 3 & 17.5 \\
\hline AV Sclerosis & 4 & 26.1 \\
\hline MV Prolapse & 6 & $100 \%$ \\
\hline Total & 23 & Wall \\
\hline
\end{tabular}

In our study, none of the patients had regurgitation lesions by Doppler ECHO or Regional Wall Motion Abnormalities. Also all the patients studied had EF $>50 \%$. 


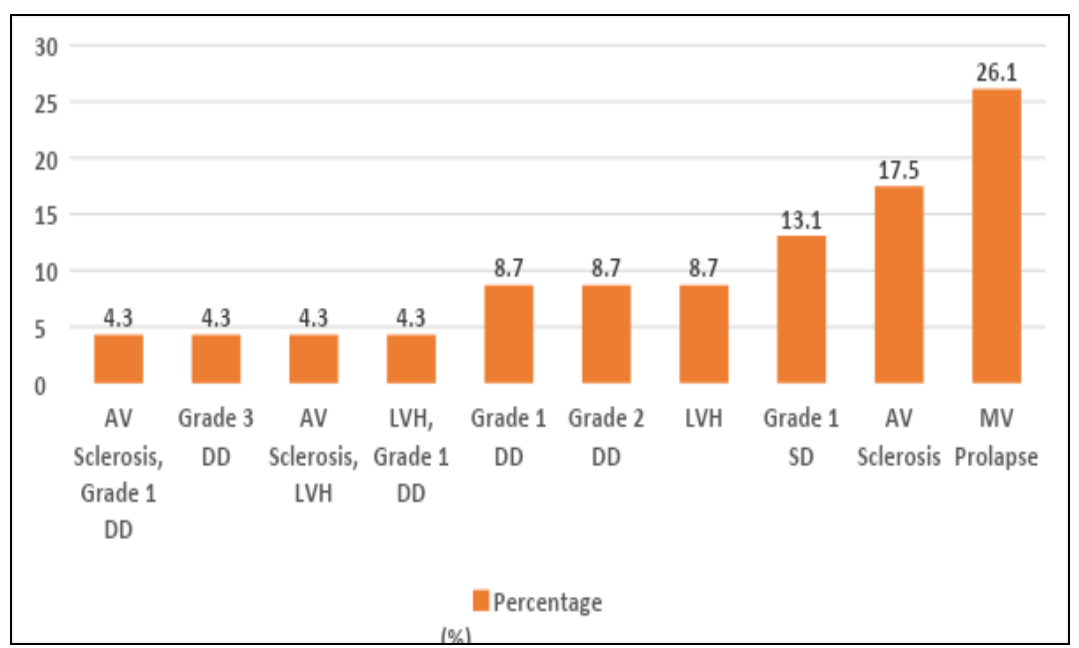

Fig 2: Distribution of study participants according to abnormal Echo findings ( $\mathrm{n}=23)$

Table 3: Distribution of study participants according to CKMB level $(\mathrm{n}=50)$

\begin{tabular}{|c|c|c|}
\hline CKMB level & Frequency & Percentage (\%) \\
\hline Normal & 30 & 60 \\
\hline Elevated & 20 & 40 \\
\hline Total & 50 & $100 \%$ \\
\hline
\end{tabular}

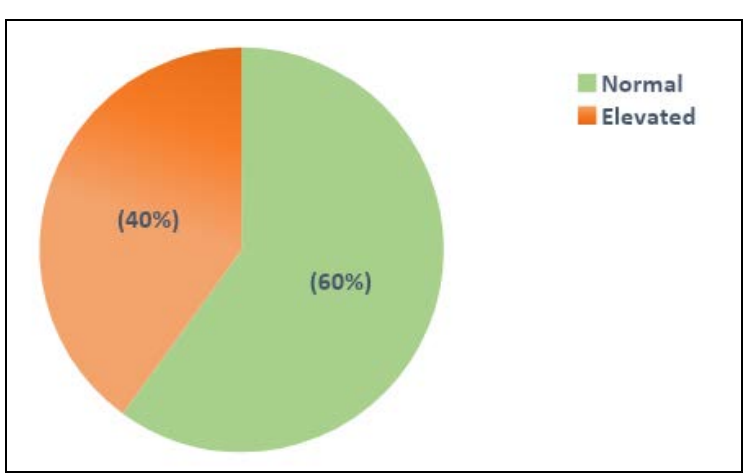

Fig 3: Distribution of study participants according to CKMB level $(n=50)$

The average CKMB among the study participants was 23.6 $\pm 8.8 \mathrm{IU} / \mathrm{l}$ (Normal value: <25 IU/L)
Table 4: Distribution of Study Participants According to TnI Level $(n=50)$ (Prevalence)

\begin{tabular}{|c|c|c|}
\hline Tn I level & Frequency & Percentage (\%) \\
\hline Normal & 32 & 64 \\
\hline Elevated & 18 & 36 \\
\hline Total & 50 & $100 \%$ \\
\hline
\end{tabular}

The average Troponin I among the study participants was $0.4 \pm 0.5$ $\mathrm{ng} / \mathrm{ml}$ (Normal value $<0.04 \mathrm{ng} / \mathrm{dl}$ )

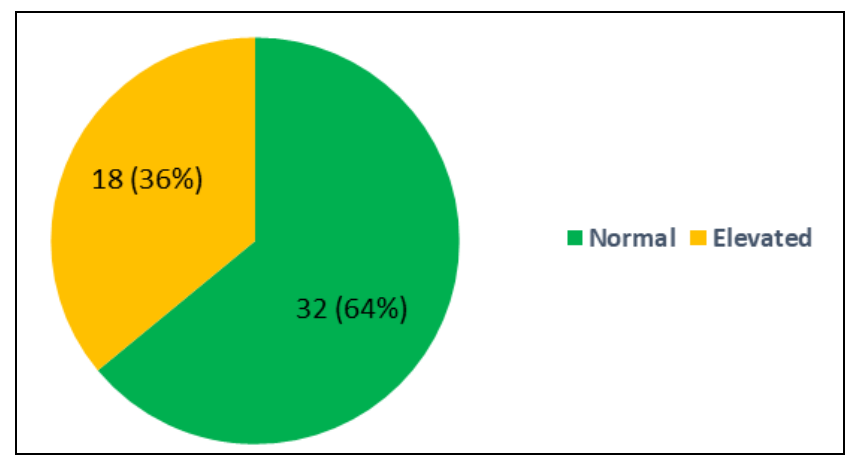

Fig 4: Distribution of study participants according to Tn I level (n $=50$ )

Table 5: Association between characteristics of study participants and Troponin I level $(n=50)$

\begin{tabular}{|c|c|c|c|c|}
\hline Characteristics & Normal Tn I level (n = 18) & Elevated Tn I level (n = 32) & Test Value & p value \\
\hline Age (in years) & $38.11 \pm 17.79$ & $49.94 \pm 17.21$ & $\mathrm{U}=169.5$ & $0.017^{*}$ \\
\hline Gender (Male) & $9(50 \%)$ & $18(56.3 \%)$ & $\chi=0.181$ & 0.67 \\
\hline Weight & $70.44 \pm 21.13$ & $72.09 \pm 13.75$ & $\mathrm{U}=277.5$ & 0.832 \\
\hline Smoking History & $4(22.2 \%)$ & $10(31.3 \%)$ & $\chi=0.466$ & 0.495 \\
\hline Alcohol History & $4(22.2 \%)$ & $6(18.8 \%)$ & $\chi=0.087$ & 0.768 \\
\hline Diabetes & $4(22.2 \%)$ & $16(50 \%)$ & $\chi=3.704$ & 0.054 \\
\hline Hypertension & $4(22.2 \%)$ & $15(46.9 \%)$ & $\chi=2.972$ & 0.085 \\
\hline Duration of tachycardia & $1.83 \pm 1.043$ & $2.75 \pm 1.16$ & $\mathrm{U}=163.0$ & $0.009^{*}$ \\
\hline Chest pain & $6(33.3 \%)$ & $18(56.3 \%)$ & $\chi=2.424$ & 0.119 \\
\hline Heart rate & $201.78 \pm 9.03$ & $211.44 \pm 15.50$ & $\mathrm{U}=175.5$ & $0.023^{*}$ \\
\hline Systolic Blood Pressure & $1289.89 \pm 22.60$ & $121.81 \pm 16.07$ & $\mathrm{U}=229.0$ & 0.229 \\
\hline Diastolic Blood Pressure & $78.44 \pm 14.03$ & $71.59 \pm 13.94$ & $\mathrm{U}=205.5$ & 0.094 \\
\hline ECG (AVRT) & $2(11.1 \%)$ & $3(9.4 \%)$ & $\chi=0.039$ & 0.844 \\
\hline CKMB & $20.17 \pm 5.85$ & $25.53 \pm 9.72$ & $\mathrm{U}=193.5$ & $0.056^{*}$ \\
\hline Haemoglobin & $12.09 \pm 2.64$ & $9.93 \pm 1.40$ & $\mathrm{U}=152.0$ & $0.006^{*}$ \\
\hline
\end{tabular}

The mean weight of the study participants was $71.5 \pm 16.6 \mathrm{~kg}$.

The mean heart rate of study participants was $207.96 \pm 14.22$ beats per minute.

The mean duration of tachycardia among the study participants was $2.42 \pm 1.19$ hours.

The average Systolic Blood Pressure among the study participants was $124.7 \pm 18.9 \mathrm{~mm} / \mathrm{Hg}$.

The average Diastolic Blood Pressure among the study participants was $74.1 \pm 14.2 \mathrm{~mm} / \mathrm{Hg}$.

The mean Haemoglobin of the study participants was $10.71 \pm 2.18 \mathrm{~g} \%$. 


\section{Discussion}

In this study, the prevalence of Troponin I level elevation in SVT patients was $36 \%$. The current, more sensitive cardiac biomarker assays like Troponin I has made it possible to determine the prognostic value of Troponin assays in patients presenting to the emergency department with SVT. It has however, been emphasized that Troponin I elevation does not necessarily indicate obstructive Coronary Artery Disease. Momentarily there is no difference in the diagnosis of Coronary Artery Disease based on Troponin elevation.

Heart rate during SVT and the presence of impaired left ventricular systolic function have been reported as predictors of troponin elevation ${ }^{6,7}$. In our study, heart rate did not correlate with elevation of Troponin I, although the duration of tachycardia had a significant correlation.

\section{Study Limitations}

- The major limitation of this study is its small sample size.

- $\quad$ Some patients refused the hospital stay after successful cardioversion, so only fewer parameters were measured for them.

- Eventually, the results of this single-centre study might not be applied to general populations because of demographic factors.

- No follow - up was carried out. Serial Troponin I measurements were not done in order to rule out Coronary Artery Disease.

- Major investigations like Angiogram or Thread Mill Test were not performed.

\section{Conclusion}

- In this study, we found that older age, lower diastolic blood pressure, longer tachycardia duration, and chest pain were the major predictors of Troponin I elevation in SVT patients

- The prevalence of elevated troponin I in patients presenting with SVT is $36 \%$.

- Furthermore it stipulates a need to carry out more studies with large sample groups and also to determine early Troponin I measurement methods in order to illuminate the significance of Troponin I in predicting the prognosis of SVT patients and in deciding the prompt treatment.

- Our study unriddles that Troponin I elevation is not pathognomic for CAD and may sometime lead to misdiagnosis.

- Patients cannot be diagnosed with Acute Coronary Syndrome, only because they have elevated Troponins. Caution is advised with the use of invasive assessments such as coronary angiography in the differential diagnosis of patients with SVT and elevated Troponins.

\section{References}

1. Schmitz G, Rezaie S. Do elevated troponins during supraventricular tachycardia predict the presence of coronary artery disease. Emergency medicine volume 3(4):1000e132

2. Miranda RC, Machado MN, Takakura IT, da Mata PF, da Fonseca CG, et al. Elevated troponin levels after prolonged supraventricular tachycardia in patient with normal coronary angiography. Cardiology 2006;106:10-13.
3. Yeo KK, Cruz L, Hong R. Tachycardia-induced elevations in cardiac troponin in the absence of coronary artery disease. Hawaii Med J 2006;65:86-87.

4. Redfearn DP, Ratib K, Marshall HJ, Griffith MJ. Supraventricular tachycardia promotes release of troponin I in patients with normal coronary arteries. Int J Cardiol. 2005;102:521-522.

5. Bukkapatnam RN, Robinson M, Turnipseed S, Tancredi D, Amsterdam E, et al. Relationship of myocardial ischemia and injury to coronary artery disease in patients with supraventricular tachycardia. Am J Cardiol. 2010;106:374-377.

6. Chow GV, Hirsch GA, Spragg DD, Cai JX, Cheng A, et al. Prognostic significance of cardiac troponin I levels in hospitalized patients presenting with supraventricular tachycardia. Medicine (Baltimore) 2010;89:141-148.

7. Ben Yedder N, Roux JF, Paredes FA. Troponin elevation in supraventricular tachycardia: primary dependence on heart rate. Can J Cardiol. 2011;27(1):105-9. 